\title{
Uso indevido de drogas entre médicos: problema ainda negligenciado
}

\author{
Substance misuse among physicians: a still neglected problem
}

Thiago Marques Fidalgo', Dartiu Xavier da Silveira'

Objetivo: Avaliar as impressões e as opiniões de médicos de áreas clínicas acerca do uso de drogas entre médicos e, mais especificamente, em ambiente cirúrgico. Métodos: Foram sorteados 100 médicos não-residentes de áreas clínicas, selecionados ao acaso entre os profissionais com vínculo em um hospital público de São Paulo. Destes, 83 concluíram o estudo, respondendo perguntas sobre o uso de drogas entre médicos. Resultados: Declararam conhecer algum colega com problemas relacionados ao uso de substâncias 67,5\% dos médicos. Esse índice foi de $41,0 \%$ quando a pergunta era acerca de drogas disponíveis em ambiente cirúrgico, visto que 68,6\% julgam ser fácil o desvio de psicotrópicos desse local. Além disso, 60,2\% acreditam que os médicos são mais suscetíveis ao uso abusivo de psicotrópicos quando comparados à população geral. No entanto, 88,0\% do total consideram difícil a procura por ajuda especializada. A porcentagem é de $56,6 \%$ dos que não conhecem serviço de atendimento direcionado exclusivamente para esses profissionais, algo que, na opinião de $83,1 \%$, facilitaria a busca por tratamento. Dos participantes, 96,4\% declararam não apresentar problemas relacionados ao uso de substâncias, ainda que 16,9\% admita já ter feito uso de psicotrópicos sem prescrição. Conclusão: $A$ freqüência de uso de psicotrópicos sem prescrição foi elevada. No entanto, parcela considerável não considera isso problema. A maioria dos profissionais não conhece serviços de atendimento específico para médicos.

\section{Palavras-chave}

Psicotrópicos, médicos, dependência.

\section{ABSTRACT}

Objective: To evaluate the opinions and attitudes about substance use among clinical physicians. Method: A hundred physicians of clinical areas were selected in a public hospital of São Paulo. All of them were asked to answer a questionnaire with some questions about drug use. $83 \%$ completed the research protocol properly. Results: $60.2 \%$ of the interviewed clinicians think that physicians are more likely to develop substance use disorders than general population. $67.5 \%$ of them stated they knew a colleague presenting a substance use disorder and in that in $41.0 \%$ of the cases the abused substance was a psychotropic available on the surgery facilities. However, $96.4 \%$ of the participants denied they could have a substance use problem, although $16.9 \%$ declared they had already used non prescribed psychotropics. Benzodiazepines were the most frequently used substances. In addition, $88.0 \%$ of them consider it was difficult to search for medical help. They added that a service exclusive for physicians would make this search easier. Nevertheless, 56.6\% are not

1 Programa de Orientação e Atendimento a Dependentes (Proad) do Departamento de Psiquiatria da Universidade Federal de São Paulo (Unifesp).

Programa de Orientação e Atendimento a Dependentes (Proad) do Departamento de Psiquiatria da Escola Paulista de Medicina (EPM) da Universidade Federal de São Paulo (Unifesp).

Endereço para correspondência: Thiago Marques Fidalgo

Rua Aureliano Coutinho, 231, apto. 91, Embaré - 11040-241 - Santos, SP

E-mail:marquesfidalgo@yahoo.com.br 


\section{Keywords}

Psychotropic, physicians, dependence. aware of the existence of such a service. Conclusion: Non-prescribed psychotropic use was high. However, most part of the clinicians does not consider this a problem. Most of the professionals do not know programs on substance use disorders specific for physicians, what would be a very positive initiative according to great part of them.
Segundo a mitologia grega, Asclépio é o deus da Medicina. Menos conhecido, no entanto, é o fato de que todos os conhecimentos de Asclépio Ihe foram ensinados por Chíron, um centauro que dominava os poderes curativos de todas as ervas, curando qualquer doença. Ele próprio, no entanto, era portador de ferida incurável, causada pela flecha de Hércules'. Eis o grande paradoxo. O curador ferido, incapaz de tratar a si mesmo. Eis o fenômeno que ainda hoje assombra todos aqueles que se dispõem a seguir os passos de Asclépio.

Hipócrates, pai da Medicina, em um de seus aforismos aconselha: "Médico, cura-te"2. Palavras sábias que ainda hoje encontram ressonância. Modernamente, segundo Olivenstein ${ }^{3}$, há um "não-dito" na medicina, caracterizado pelo medo-ódio cuja principal fonte é o medo da própria morte. Corroborando isso, a literatura médica atual tem como consenso que médicos constituem grupo vulnerável a uma série de doenças, tanto físicas quanto mentais. É sobre esse último aspecto que repousa nosso interesse.

O editorial publicado em 1903, no JAMA4 ${ }^{4}$ já apontava para a necessidade de estudo detalhado dos aspectos psíquicos dos profissionais médicos. Desde então, inúmeros levantamentos foram realizados objetivando a compreensão plena desse fenômeno. Infelizmente, novo editorial publicado cem anos depois, no mesmo periódico, afirma que pouco foi feito para mudar tal situação ${ }^{5}$. Suicídio ${ }^{6-8}$, transtornos do humor e de ansiedade ${ }^{9,10}$, além de dependência química ${ }^{11-13}$ são encontrados em taxas sempre muito mais elevadas entre profissionais da saúde, quando comparados com a população geral. Isso adquire importância ainda maior quando se considera que tais patologias apresentam enorme impacto não só na qualidade técnica do atendimento oferecido, mas, também, no tipo de relação médico-paciente que se estabelece ${ }^{14}$. Martins $s^{15}$, em estudo de referência no meio médico-hospitalar, relata que entre os mecanismos adaptativos utilizados pelos médicos para lidar com o estresse encontram-se: a falta de empatia em relação às queixas dos pacientes, prejudicando, assim, o adequado diagnóstico e a terapêutica; a utilização da ironia e do humor negro, que acabam por contaminar também as relações pessoais do profissional; e, a falta de cuidados em relação à própria saúde, uma vez que o médico-paciente nega sua condição de doente, escondendo suas dificuldades emocionais dos colegas, da família e de si mesmo ${ }^{14}$.

Por tudo isso, torna-se fundamental a realização de estudos que se aprofundem nessa questão em cada contexto específico, de maneira que ações de suporte e preventivas possam ser planejadas e implementadas, a fim de assegurar a qualidade da assistência oferecida e da saúde integral do médico. Dessa forma, objetivou-se realizar estudo preliminar descritivo para avaliar as impressões e as opiniões de médicos de áreas clínicas acerca do uso de drogas entre médicos e, mais especificamente, em ambiente cirúrgico.

\section{MÉTODOS}

Foram identificados 100 médicos de especialidades clínicas, selecionados de maneira aleatória entre os profissionais com algum tipo de vínculo em um hospital público de São Paulo. Do total de médicos, 83 (83,0\%) concluíram o estudo. Todos responderam a diversos instrumentos validados no meio médico-hospitalar para detecção de transtornos psiquiátricos, além de questionário acerca de suas impressões e percepções sobre o uso de substâncias psicotrópicas no meio médico-hospitalar. Foram necessários, em média, 40 minutos para responder a todas as perguntas. Todas as respostas foram organizadas em um banco de dados, utilizando o software SPSS 13.0. Esta pesquisa é parte do projeto aprovado pelo Comitê de Ética da Universidade Federal de São Paulo (Ref.:\#.1267/04).

\section{RESULTADOS}

A partir dos dados coletados, traçou-se o perfil sociodemográfico da população estudada, conforme disposto na Tabela 1. No Gráfico 1, encontra-se a divisão por especialidades dos médicos de áreas clínicas incluídos no estudo.

Quanto ao uso de substâncias, 96,4\% dos participantes do estudo declararam não apresentar problemas relacionados ao uso de psicotrópicos, embora 16,9\% admitam já ter feito uso de algum deles sem prescrição ou acompanhamento adequados (com destaque para os benzodiazepínicos). Além disso, $60,2 \%$ dos entrevistados acreditam que os médicos são mais suscetíveis ao uso abusivo de psicotrópicos quando comparados à população geral, e 67,5\% dos médicos declararam conhecer algum colega com problemas relacionados ao uso de substâncias. Esse índice foi de $41,0 \%$ quando a pergunta era acerca do abuso de drogas disponíveis em ambiente 
Tabela 1. Dados sociodemográficos de médicos clínicos de um hospital público da cidade de São Paulo $(n=83)$.

\begin{tabular}{|c|c|}
\hline & Médicos clínicos \\
\hline \multicolumn{2}{|l|}{ Sexo (\%) } \\
\hline Masculino & 51,8 \\
\hline Feminino & 48,2 \\
\hline Idade em anos (média \pm desvio-padrão) & $38,6 \pm 8,8$ \\
\hline \multicolumn{2}{|l|}{ Estado civil (\%) } \\
\hline Solteiros & 30,1 \\
\hline Casados & 60,2 \\
\hline \multicolumn{2}{|l|}{ Graduação (\%) } \\
\hline Universidade pública & 65,0 \\
\hline Universidade particular & 35,0 \\
\hline \multicolumn{2}{|l|}{ Vínculo com o hospital (\%) } \\
\hline Pós-graduando & 37,3 \\
\hline Contratado & 15,7 \\
\hline Concursado & 36,1 \\
\hline Outros & 10,8 \\
\hline Carga horária semanal em horas (média \pm desvio-padrão) & $55,3 \pm 15,6$ \\
\hline Exercício de outra atividade profissional (\%) & 69,9 \\
\hline \multicolumn{2}{|l|}{ Atividade de lazer (\%) } \\
\hline Nunca & 11,3 \\
\hline 1 vez na semana & 26,3 \\
\hline 2 vezes na semana & 11,3 \\
\hline 3 vezes na semana & 13,8 \\
\hline 4 vezes na semana & 6,3 \\
\hline 5 vezes na semana ou mais & 6,3 \\
\hline
\end{tabular}

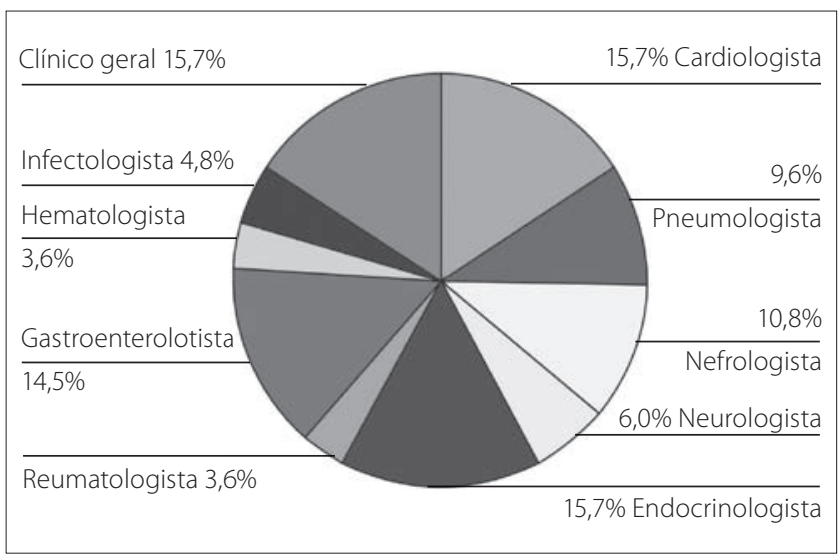

Gráfico 1. Distribuição por especialidades de médicos clínicos de um hospital público da cidade de São Paulo $(n=33)$.

cirúrgico. Em complementação a tal dado, 68,6\% dos clínicos julgam ser fácil o desvio de psicotrópicos desse local.

Em relação à busca por tratamento, $88,0 \%$ do total consideram difícil a procura por ajuda especializada. Entre os fatores apontados para tanto tem-se o medo de discriminação (segundo 38,6\% dos clínicos), o medo de represálias no trabalho $(28,9 \%)$ e a dificuldade em admitir o problema (20,5\%). Tal episódio é agravado pelo fato de 56,6\% dos médicos não conhecerem serviço de atendimento direcionado exclusivamente para esses profissionais, algo que, na opinião da maioria (83,1\%), facilitaria a busca por tratamento.

\section{DISCUSSÃO}

A freqüência de uso de psicotrópicos sem prescrição foi elevada, embora parcela considerável dos médicos não considere isso problema. Além disso, a maioria dos profissionais não conhece serviços de atendimento específicos para médicos com problemas relacionados ao uso de substâncias, o que, na opinião de quase todos os entrevistados, facilitaria a busca por ajuda. Esses achados reforçam a necessidade do desenvolvimento de programas de suporte psiquiátrico voltados especificamente para esses profissionais.

Os médicos deveriam, acima de tudo, buscar a construção de rotina pautada pela qualidade de vida, com equilíbrio entre as horas trabalhadas e as horas dispensadas ao lazer e à higiene mental. Assim, estarão mais aptos a exercer suas atividades com menor risco de falhas técnicas e com maior disponibilidade, resultando o estabelecimento da relação médico-paciente mais satisfatória.

\section{CONCLUSÃO}

A freqüência de uso de psicotrópicos sem prescrição foi elevada. No entanto, parcela considerável não considera isso problema. A maioria dos profissionais não conhece serviços de atendimento específico para médicos.

\section{REFERÊNCIAS}

1. De Marco M, organizador. A face humana da Medicina. São Paulo: Casa do Psicólogo; 2003.

2. Benetton LG. Temas de psicologia em saúde - a relação profissional-paciente. São Paulo: Ed. L. G. Benetton; 2002

3. Olivenstein C. O não-dito das emoções. São Paulo: Summus; 1988

4. Editorial - Suicides of physicians and the reasons. JAMA. 1903;41:263-4.

5. Editorial - Suicides of physicians and the reasons. JAMA. 2003 Jul;290:414

6. Mawardi BH. Satisfaction, dissatisfactions and causes of stress in medical practice. JAMA. 1979;241:1483-6

7. Evaus BJ, Stanley RO, Mestrovic R, Rose L. Effects of comunication skilss training on student diagnostic efficiency. Medical Education. 1991;25:517-26.

8. Meleiro AM. Suicídio entre médicos e estudantes de Medicina. Rev Assoc Med Brasileira. 1998:44(2):135-40.

9. Nogueira-Martins LA, Jorge MR. Natureza e magnitude do estresse na residência médica. Rev Assoc Med Bras. 1998:44(1):28-34

10. FrankE, Dingle AD. Self-reported depression and suicide attempts among US women physicians. Am J Psychiatry. 1983;1887-94.

11. Niel M. Anestesistas e uso de drogas: um estudo qualitativo [dissertação]. São Paulo: Escola Paulista de Medicina da Universidade Federal de São Paulo; 2003.

12. Booth JV, et al. Substance abuse among physicians: a survey of academic anesthesiology programs. Anesthesia \& Analgesia. 2002;95(4):10240-30.

13. Modlin HC, Montes A. Narcotic addiction in physicians. Am J Psychiatry. 1964;121:358-65.

14. Martins LAN. Programa de atenção à saúde e qualidade de vida do médico. Conselho Federal de Medicina, Brasil; 2004.

15. Martins LAN. Atividade médica: fatores de risco para a saúde mental do médico. Rev Bras Clin Terapêutica. 1991;20(9):355:64. 